\begin{tabular}{|c|l|}
\hline Title & Fabrication of semiconductor Kagome lattice structure by selective area metal organic vapor phase epitaxy \\
\hline Author(s) & Mohan, Premila; Nakajima, Fumito; A kabori, Masashi; Motohisa, Junichi; Fukui, Takashi \\
\hline Citation & $\begin{array}{l}\text { A pplied Physics Letters, 83(4), 689-691 } \\
\text { https://doi.org/40.1063/1593823 }\end{array}$ \\
\hline Issue Date & $2003-07-28$ \\
\hline Doc URL & http://hdl.handle.net/2115/5519 \\
\hline Rights & Copyright $\odot 2003$ A merican Institute of Physics \\
\hline Type & article \\
\hline File Information & APL83-4.pdf \\
\hline
\end{tabular}

Instructions for use 


\title{
Fabrication of semiconductor Kagome lattice structure by selective area metalorganic vapor phase epitaxy
}

\author{
Premila Mohan, a) Fumito Nakajima, Masashi Akabori, Junichi Motohisa, \\ and Takashi Fukui \\ Research Center for Integrated Quantum Electronics (RCIQE), Hokkaido University, North 13 West 8, \\ Sapporo 060-8628, Japan
}

(Received 10 March 2003; accepted 21 May 2003)

\begin{abstract}
Artificial two-dimensional semiconductor Kagome lattice structures formed by quantum wires can show ferromagnetism when the flatband is half filled, even though it does not have any magnetic elements. Experimental realization of such a Kagome lattice structure is reported. The structure, with different pattern periods, was formed with GaAs quantum wires by selective area metalorganic vapor phase epitaxy on GaAs (111)B substrates. To overcome the lateral overgrowth and to improve the shape of smaller period pattern, flow rate modulation epitaxy was employed and a GaAs Kagome lattice structure with $1 \mu \mathrm{m}$ period was effectively grown. () 2003 American Institute of Physics. [DOI: 10.1063/1.1593823]
\end{abstract}

In the recent years, quantum dots have attracted considerable interest because of their novel device applications in fields such as optics, optoelectronics, quantum computing, etc. Also, their atom-like properties make them a good candidate for studying the physics of confined carriers and many-body effects. Quantum dots are also referred as "artificial atoms" because of their discrete energy spectrum. They have great flexibility because their properties can be artificially engineered, thereby facilitating the emergence of new research directions. Because of their unique properties quantum dots can be used as building blocks for novel and flexible nanostructures such as artificial molecules. Oosterkamp et al. have created such an artificial molecule and have demonstrated how it undergoes transformation from something like an ionic to an analog of the covalent state when excited by microwave radiation. ${ }^{1}$ Extending this idea further, artificial lattices can also be formed with quantum dots as building blocks. Such an idea of using quantum dots to form an artificial two-dimensional lattice is very promising since any type of lattice structure that cannot be realized in real materials can be designed. The advancement in nanotechnology has led to the proposal of various theoretical ideas for semiconductor two-dimensional lattice patterns with possible interesting effects.

A Kagome lattice is composed of corner-shared triangles and they are the most geometrically frustrated twodimensional lattice, but unfortunately there are no physical systems that adopt the lattice. The interesting feature is that it has dispersionless flatbands in its single particle band structure. It has been theoretically proven that ferromagnetism appears in such a lattice when the flatband is half filled. ${ }^{2}$ Recently, it has been predicted that semiconductor quantum dots can be used as building blocks to fabricate the Kagome lattice structure. ${ }^{3}$ The formation of such an artificial Kagome lattice using quantum dots is very promising and the appearance of ferromagnetism in such a nonmagnetic semiconduc-

\footnotetext{
a) Author to whom correspondence should be addressed; electronic mail: pmohan@rciqe.hokudai.ac.jp
}

tor surface is a fascinating phenomenon and the experimental realization may revolutionize the electronic device industry as they can replace the magnetic materials which are not compatible with conventional integrated circuit technology.

Recently, Shiraishi, Tamura, and Takayanagi have presented a first-principle design of a semiconductor Kagome lattice made up of nonmagnetic quantum dots. ${ }^{4}$ It has been shown that a network of quantum wires effectively acts as a Kagome lattice where electrons are well localized at the cross points of two wires since the effective width of the quantum wires at the cross points is larger than the normal width of the wire. The proposed structure consisted of InAs quantum wires surrounded by InGaAs barrier regions. The width of each quantum wire was assumed to be $0.104 \mu \mathrm{m}$ and the lateral size of each two-dimensional unit cell was $0.72 \mu \mathrm{m}$. In this case, the energy separation between up- and down-spin bands was $0.05 \mathrm{meV}$ which is sufficient to experimentally observe ferromagnetic properties at low temperature. Various theoretical studies have been carried out on the semiconductor Kagome lattice structure, but to date there is no experimental realization of the same. Hence, the present work was aimed at fabricating the semiconductor Kagome lattice structure.

Selective area metalorganic vapor phase epitaxy (SAMOVPE) is the most promising method to realize such a complicated structure without process induced damage or contamination. ${ }^{5}$ Hence, in the present work, SA-MOVPE was utilized to fabricate the Kagome lattice structure. GaAs (111)B substrates are usually employed for the fabrication of two-dimensional photonic crystals whose basic structure typically consists of an array of pillars or holes. This is because $\{-110\}$ vertical facets appear during selective area growth and also the threefold symmetry on the (111)B plane facilitates the growth of triangular or hexagonal structures. ${ }^{6}$ In the present case also, GaAs (111)B was chosen as the substrate for the same reasons. This letter reports the experimental progress towards the realization of the semiconductor two-dimensional Kagome lattice structure using GaAs quantum wires on GaAs (111)B substrate by SA-MOVPE. 


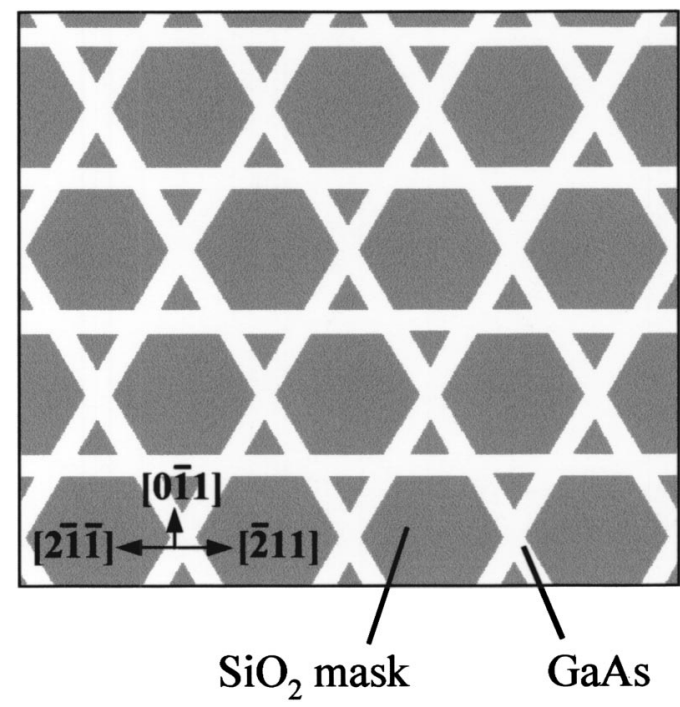

FIG. 1. Schematic illustration of the mask pattern for selective area MOVPE growth of Kagome lattice structure.

Schematic illustration of the pattern of the masked substrate used for the selective area growth is shown in Fig. 1. The mask patterns were formed on $\mathrm{SiO}_{2}$ coated GaAs (111)B substrates by electron beam lithography and wet chemical etching. Kagome lattice patterns were formed with various periods of 3,1.5 and $1 \mu \mathrm{m}$ and with wire opening widths ranging from 150 to $70 \mathrm{~nm}$. A low-pressure, horizontal, rfheated, MOVPE system was used for the selective area growth. The source materials were trimethylgallium (TMGa), trimethyaluminium (TMAl) and 20\% arsine $\left(\mathrm{AsH}_{3}\right)$ in $\mathrm{H}_{2}$. The partial pressure of $\mathrm{AsH}_{3}$ was 5 $\times 10^{-5}$ atm because low As coverage conditions are necessary for MOVPE growth of GaAs (111)B surface. For the growth of GaAs layers the partial pressure of TMGa was $7.3 \times 10^{-7} \mathrm{~atm}$ and for the AlGaAs barrier layers the partial pressures of TMGa and TMAl were $3.7 \times 10^{-7}$ and 1.2 $\times 10^{-6} \mathrm{~atm}$, respectively. The growth temperature was $850^{\circ} \mathrm{C}$.

GaAs/AlGaAs quantum wells were grown on the masked substrates with the above conditions to form the Kagome lattice structure. The layer structure typically consisted of a $100 \mathrm{~nm}$ GaAs buffer layer, a $20 \mathrm{~nm}$ bottom AlGaAs barrier layer, a $10 \mathrm{~nm}$ GaAs well layer, $20 \mathrm{~nm}$ top $\mathrm{AlGaAs}$ barrier layer and a $10 \mathrm{~nm}$ GaAs cap layer.

Figure 2(a) shows the scanning electron microscope (SEM) image of $3 \mu \mathrm{m}$ period Kagome lattice structure grown by SA-MOVPE. The results indicate that it is possible to realize uniform semiconductor Kagome lattice structure on GaAs (111)B substrate. However, growth on shorter period pattern did not exactly follow the mask pattern and shape deterioration was found to occur due to lateral overgrowth (LOG) on the mask area [Figs. 2(b) and 2(c)]. In general, LOG can be overcome by optimizing the growth conditions. Apart from the growth conditions, the lateral overgrowth significantly depends on the pattern fill ratio, which is the ratio of the pattern opening area to the total area. It has been reported that by choosing the exact pattern fill ratio it is possible to reduce the LOG in photonic crystals. ${ }^{6}$ But unlike a photonic crystal mask pattern, the Kagome lattice pattern consists of an array of two kinds of $\mathrm{SiO}_{2}$ masked regions that are different in both shape as well as size, i.e., it has an array of hexagonal mask areas surrounded by triangular mask areas, and the size of the triangular mask area is less than one-sixth of the hexagonal area. The presence of this smaller triangular mask area is very critical since it restricts the increase of the fill ratio. Each pattern period has a critical fill ratio beyond which it cannot be increased since increasing the wire opening area will lead to the disappearance of smaller triangles. Unlike photonic crystals, in which a small percentage of LOG does not significantly affect the end result, the occurrence of LOG is a more serious problem in the case of a Kagome lattice structure since even a small percentage of LOG will bury the smaller triangular mask area resulting in a completely different pattern.

The pattern shape also plays a significant role in the occurrence of LOG. In the Kagome lattice mask pattern, the opening area forms the Kagome lattice structure and, as already mentioned, the $\mathrm{SiO}_{2}$ mask consists of hexagons surrounded by triangles. Around these hexagonal and triangular masks the opening area has six and three corners, respectively. The presence of these corners plays a significant role in the shape deterioration since these corners consist of many steps and kinks. Since $\mathrm{Ga}$ atoms have a longer diffusion length than $\mathrm{Al}$ atoms, the migrating $\mathrm{Ga}$ atoms will be incorporated into these steps and kinks and hence blunting the sharp corners. This incorporation probability of $\mathrm{Ga}$ is related to the density of the dangling bonds at the step edge, and the dangling bond density is related to the availability of As at the step edge. ${ }^{7}$ Hence, low As coverage is the key factor to ensure that the migrating $\mathrm{Ga}$ atoms are not incorporated into steps and kinks at the corners and thereby preventing lateral overgrowth.

The origin of shape deterioration of the grown Kagome lattice pattern can be explained as follows. Due to LOG the hexagonal mask area assumed a triangular shape as shown in Fig. 3. This is because the $\langle 2-1-1\rangle$ corners and $\langle-211\rangle$
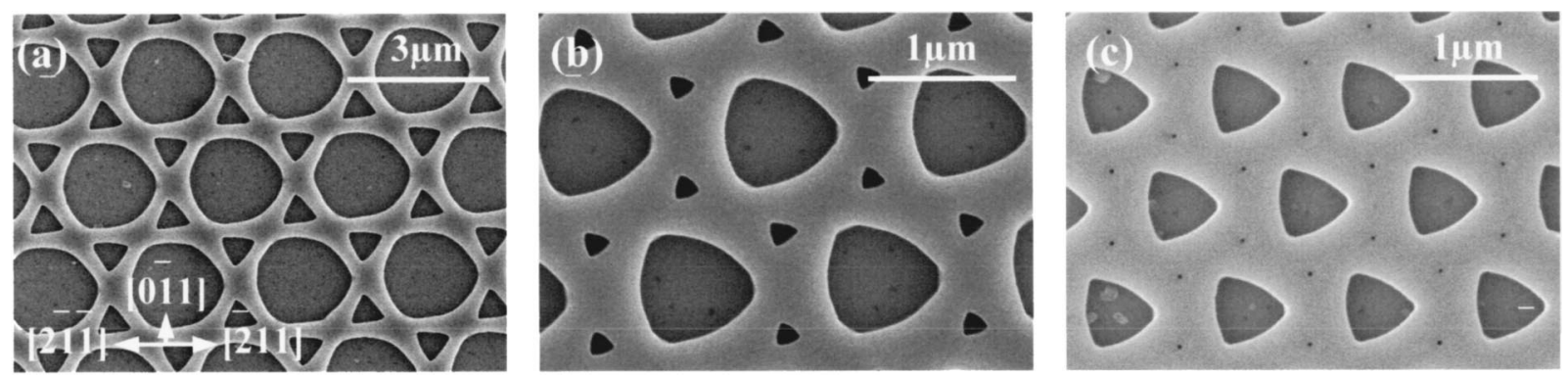

FIG. 2. SEM images of SA-MOVPE grown Kagome lattice structure with periods (a) $3 \mu \mathrm{m}$ (b) $1.5 \mu \mathrm{m}$ and (c) $1 \mu \mathrm{m}$ 


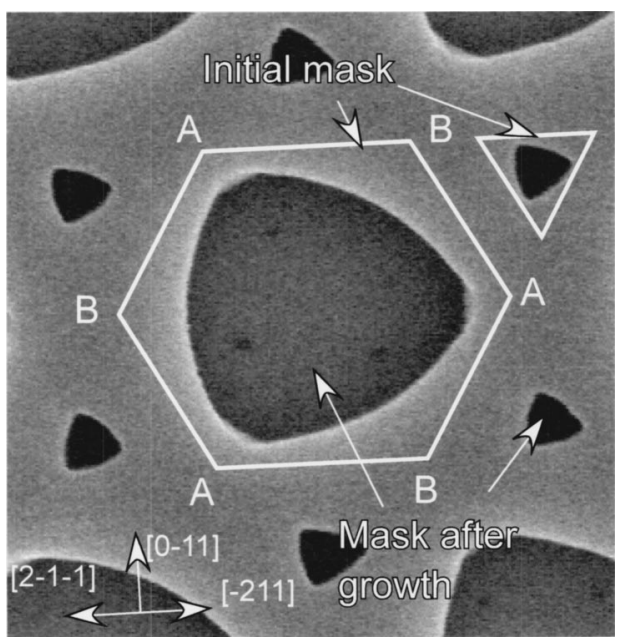

FIG. 3. Schematic illustration of the structural deterioration of the Kagome lattice pattern.

corners are crystallographically different and LOG is predominant in the three corners which face the $\langle-211\rangle$ direction (B direction), while LOG is less pronounced at other corners, which face the $\langle 2-1-1\rangle$ direction (A direction). Under low As coverage growth condition $\{110\}$ and $\{111\} \mathrm{A}$ facets preferentially appear and $\{111\}$ A facets can be formed in the A direction. Therefore, as a result of facet formation, step flow growth at $\langle 2-1-1\rangle$ corners can be suppressed. However, no formation of stabilized facets occurs in the B direction, and hence it is impossible to suppress step flow growth and hence LOG. In the case of the triangular mask area, even though it retained the triangular shape in spite of LOG, a significant change in orientation was noticed. A similar explanation can be given in this case also. LOG being predominant in the B direction and indistinct in the A direction results in the change of orientation. Hence, in order to obtain uniform Kagome lattice structure, suppression of LOG in both directions is needed.

Another important observation was that the LOG increases as the pattern period decreases (Fig. 2). This is because in smaller period patterns, the mask area is small and hence the probability of migration of group III species from the $\mathrm{SiO}_{2}$ mask area to the opening area is greater than the desorption rate of excess $\mathrm{Ga}$ and so the LOG is larger resulting in reduction of the mask area. Thus, the effective mask area becomes still smaller as growth proceeds and LOG on mask area is enhanced.

Hence, to improve the shape of the smaller period patterns, flow rate modulation epitaxy (FME), which is based on alternate supply of group III and group V sources, was employed. It has been proved that FME has a remarkable effect on improving the shape controllability in SA-MOVPE. ${ }^{8}$ The flow sequence consisted of alternate TMGa/TMAl and $\mathrm{AsH}_{3}$ flow with a $\mathrm{H}_{2}$ purge period in between in order to avoid gas mixing. The two AlGaAs barrier layers and the GaAs quantum well layer were grown by FME and the GaAs buffer layer and the GaAs capping layer were grown by conventional MOVPE. The growth temperatures for MOVPE and

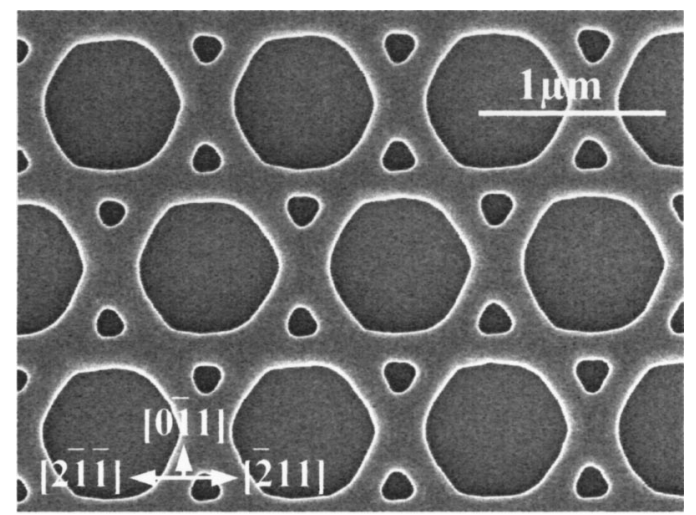

FIG. 4. SEM image of $1 \mu \mathrm{m}$ Kagome lattice structure grown with optimized conditions.

FME were 850 and $750{ }^{\circ} \mathrm{C}$, respectively. By using FME growth, the shape of the grown layer was found to follow the mask pattern and the edges were found to be sharp, indicating that the migrating $\mathrm{Ga}$ atoms are not incorporated into steps and kinks at the concave positions. This is probably because a low As coverage during the TMGa/TMAl supply period in FME prevents step-flow growth. Even though the mask shape had sharp edges the surface morphology was not smooth due to hillocks produced by three-dimensional nucleation. The As trimers formed on the surface should evaporate during the purging time after supplying $\mathrm{AsH}_{3}$ so as to obtain mass-transport-limited growth. However, the purge time of 1 $\mathrm{s}$ is too small to complete the evaporation of As trimers at $750^{\circ} \mathrm{C}$. Hence, FME growth was carried out by changing the purging time from 1 to $2 \mathrm{~s}$. The $\mathrm{H}_{2}$ purge of $2 \mathrm{~s}$ after TMGa/ TMAl flow also enhances the desorption of excess group III species facilitating the suppression of LOG. Each sample almost kept its initial shape after growth (Fig. 4). Therefore, the results indicate that it is possible to fabricate Kagome lattice structure with smaller period patterns by SA-MOVPE.

In summary, semiconductor Kagome lattice structures of $1 \mu \mathrm{m}$ period were grown by selective area metalorganic vapor phase epitaxy on GaAs (111)B substrates. The results obtained are very promising and can be extended to the growth of Kagome lattice structure with still smaller periods for experimental observation of ferromagnetism. Further investigations are under way in fabricating such smaller structures.

${ }^{1}$ T. H. Oosterkamp, T. Fujisawa, W. G. van der Wiel, K. Ishibashi, R. V. Hijman, S. Troche, and L. P. Kouwenhoven, Nature (London) 395, 873 (1998).

${ }^{2}$ A. Mielke, J. Phys. A 24, L73 (1991).

${ }^{3}$ H. Tamura, K. Shiraishi, T. Kimura, and H. Takayanagi, Phys. Rev. B 65, 085324 (2002).

${ }^{4}$ K. Shiraishi, H. Tamura, and H. Takayanagi, Appl. Phys. Lett. 78, 3702 (2002).

${ }^{5}$ T. Fukui, S. Ando, T. Honda, and T. Toriyama, Surf. Sci. 267, 236 (1992).

${ }^{6}$ M. Akabori, J. Takeda, J. Motohisa, and T. Fukui, Physica E (Amsterdam) 13, 446 (2002).

${ }^{7}$ H. Asai, J. Cryst. Growth 80, 425 (1987).

${ }^{8}$ S. Ando, N. Kobayashi, H. Ando, and Y. Horikoshi, J. Cryst. Growth 170, 719 (1997). 